\title{
Apoio ao ensino de análise e projeto de software usando a plataforma Arduino
}

\author{
Bruno Lima, Guilherme Mattiello, Luis Galonetti, Murylo Lopes, \\ Rhuan Costa, Andre R. Ortoncelli, Paulo Nardi, Andre T. Endo \\ ${ }^{1}$ Universidade Tecnológica Federal do Paraná (UTFPR), Brasil
}

\begin{abstract}
The teaching of software analysis and design can take advantage of more specific systems for engineering courses, since some of these areas focus on embedded systems and hardware components associated. In this context, this paper presents the development of an open educational resource to support the teaching of software analysis and design using the Arduino platform. The resource consists of Arduino projects with circuit schematics and UML diagrams, helping the comprehension of requirements and the project assembly. Such resource was evaluated with Computer Engineering students, who reproduced the projects and gave feedback on the generated artifacts aiming to reach the proposed objectives. Through a survey, we observed that students were satisfied with the process and the artifacts available for the understanding and replication of the projects.
\end{abstract}

Resumo. $O$ ensino de análise e projeto de software pode usufruir de sistemas mais específicos para cursos de engenharia, pois algumas dessas áreas focam em sistemas embarcados e componentes de hardware associados. Neste contexto, este artigo apresenta o desenvolvimento de um recurso educacional aberto para apoiar o ensino de análise e projeto de software usando a plataforma Arduino. O recurso é composto por projetos desenvolvidos para a plataforma Arduino, com esquemáticos de circuitos eletrônicos e diagramas UML auxiliando a compreensão dos requisitos e montagem do projeto. Tal recurso foi avaliado com alunos do curso de Engenharia da Computação, que reproduziram os projetos e deram feedback sobre os artefatos gerados visando alcançar os objetivos propostos. Por meio de um questionário, foi observado que os alunos ficaram satisfeitos com o processo e com os artefatos disponibilizados para o entendimento e execução dos projetos.

\section{Introdução}

Disciplinas relacionadas à Engenharia de Software têm uma natureza essencialmente prática, fato que conflita diretamente com a maneira como esses conteúdos são geralmente abordados em cursos de graduação, que é predominantemente teórica [Souza et al. 2010]. Apesar do embasamento teórico ser essencial, por si só, não é suficiente para que os alunos estejam preparados para o mercado de trabalho [Damian et al. 2006].

Uma forma de amenizar esse problema é fazer com que os alunos desenvolvam pequenos projetos fictícios ou reais [Yamaguti 2016]. Porém em grande parte, tais projetos possuem ênfase em sistemas de informação e jogos [Larman 2012] - domínios que não representam o foco de alguns dos cursos de Bacharelado em Engenharia, tais como 
as Engenharia Elétrica, de Computação e de Automação, que envolvem essencialmente projetos de sistemas embarcados e componentes de hardware associados.

Nestes cursos, é comum o uso de sistemas e componentes baseados no Arduino para o apoio ao ensino de disciplinas como Programação [Brock et al. 2009, Melo et al. 2014, Mattos et al. 2015, Avila 2016], Robótica [Pinto et al. 2012, Grover et al. 2014], Probabilidade e Estatística [Cavalcante et al. 2014] e Física [de Souza et al. 2011]. No entanto, há poucos trabalhos na literatura que objetivam o apoio ao ensino de Engenharia de Software aplicada a esses cursos. Como exemplo, [Duschl et al. 2014] examinam defeitos em diagramas UML (Linguagem de Modelagem Unificada) produzidos por alunos de Engenharia Mecânica.

Neste artigo, é proposto o uso da plataforma Arduino para o processo de ensino e aprendizagem de Análise e Projeto Orientado a Objetos (APOO). Esta plataforma é bastante difundida nos cursos de Engenharia devido a seu baixo custo e popularidade na comunidade DIY (Do It Yourself). Além disso, possibilita o desenvolvimento/prototipagem de sistemas embarcados de forma simplificada [Arduino 2017]. Três contribuições são apresentadas nesse artigo: $(i)$ a introdução de uma abordagem para o ensino e aprendizagem de APOO em cursos de Engenharia; (ii) um recurso educacional que é disponibilizado de forma aberta e composto por vários projetos com artefatos relacionados (diagramas UML, esquemáticos de circuito e código fonte); e, (iii) uma avaliação e análise da abordagem introduzida.

O artigo está dividido da seguinte forma. Na Seção 2, é detalhado o recurso educacional apresentado neste trabalho por meio de um exemplo. Na Seção 3, é apresentada uma abordagem de aplicação do recurso educacional. O instrumento de avaliação do recurso, juntamente com os resultados obtidos, são apresentados na Seção 4. Por fim, na Seção 5, são apresentadas as conclusões e trabalhos futuros.

\section{Recurso Educacional}

Um recurso educacional é todo e qualquer material, equipamento ou tecnologia educacional utilizado em um processo de ensino, visando incentivar e aproximar o aluno do conteúdo [Freitas 2009]. Neste trabalho, o recurso educacional proposto consiste em um conjunto de projetos nos quais a plataforma alvo é o Arduino.

O objetivo de cada projeto é permitir que o estudante treine sua habilidade de interpretar os artefatos da UML e, a partir de sua interpretação, possa embarcá-lo no Arduino. Uma vez treinados nesta habilidade, os estudantes são incentivados a criarem seus próprios projetos e artefatos. Como resultado destas atividades, é esperado que o recurso auxilie o aluno na consolidação do conhecimento adquirido em aulas teóricas com o treinamento e na aquisição de novos conhecimentos com a criação de novos projetos.

Para cada projeto, foram gerados seis artefatos: (i) o diagrama de casos de uso; (ii) o diagrama de atividades; (iii) o diagrama de máquinas de estados; (iv) o diagrama de classe; (v) o esquemático de montagem; e (vi) o código fonte em linguagem $\mathrm{C} / \mathrm{C}++$, implementado utilizando a Arduino $\mathrm{IDE}^{1}$, pronto para ser compilado e embarcado na placa Arduino. As análises e projetos disponíveis foram documentados com diagramas

\footnotetext{
${ }^{1}$ https://www.arduino.cc/en/Main/Software
} 
UML e desenvolvidos por meio do software Astah $^{2}$. Os esquemáticos foram gerados no Fritzing ${ }^{3}$ para facilitar a montagem física dos circuitos eletrônicos, identificando portas, conexões e componentes utilizados. Atualmente, o recurso educacional proposto possui 15 projetos, disponíveis em um repositório online ${ }^{4}$. Tal repositório pode ser estendido com novos projetos e fornece os elementos necessários para a replicação deste estudo.

Nesta seção, é apresentado um dos projetos do recurso educacional, que consiste na simulação de dois semáforos, cujas luzes são representadas por LEDs de três cores (assumindo um cruzamento de vias): dois vermelhos, dois amarelos e dois verdes. No projeto, foram implementados dois semáforos e dois botões: $(i)$ um para ligar e desligar e (ii) outro para entrar/sair do modo alerta (piscando o LED amarelo). Na Figura 1, é ilustrado o esquemático para a montagem do projeto de simulação de semáforos. Tal artefato auxilia diretamente na montagem física do projeto, indicando as ligações elétricas dos componentes utilizados.

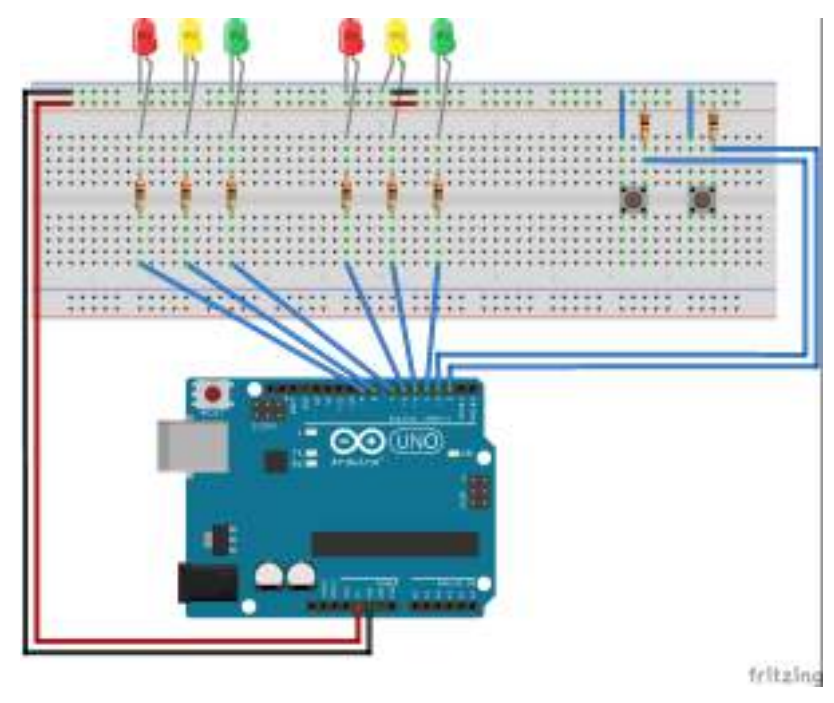

Figura 1. Esquemático - Semáforo

Nas Figuras 2, 3, 4 e 5, são ilustrados os diagramas de atividades, casos de uso, classe e máquina de estados, os quais visam o entendimento do projeto a ser reproduzido pelos alunos. Tais diagramas representam a estrutura e o comportamento, fornecendo visões estáticas e dinâmicas do sistema.

\section{Procedimentos Metodológicos}

O recurso educacional foi avaliado quanto a capacidade de auxiliar na consolidação e aquisição de conhecimentos e a sua aceitação pelos alunos. A avaliação se deu pela aplicação dos artefatos em sala de aula, em três turmas do sexto semestre do curso de Engenharia de Computação, durante a disciplina de Engenharia de Software. Os alunos possuem conhecimento prévio na linguagem de programação C e em orientação a objetos, bem como conceitos introdutórios de UML. Para cada turma, a avaliação aconteceu em seis aulas de cinquenta minutos cada. Os alunos foram aleatoriamente divididos em

\footnotetext{
${ }^{2} \mathrm{http}: / /$ astah.net/editions/community

${ }^{3}$ http://fritzing.org/home

${ }^{4}$ https://github.com/andreendo/umlino-rea
} 


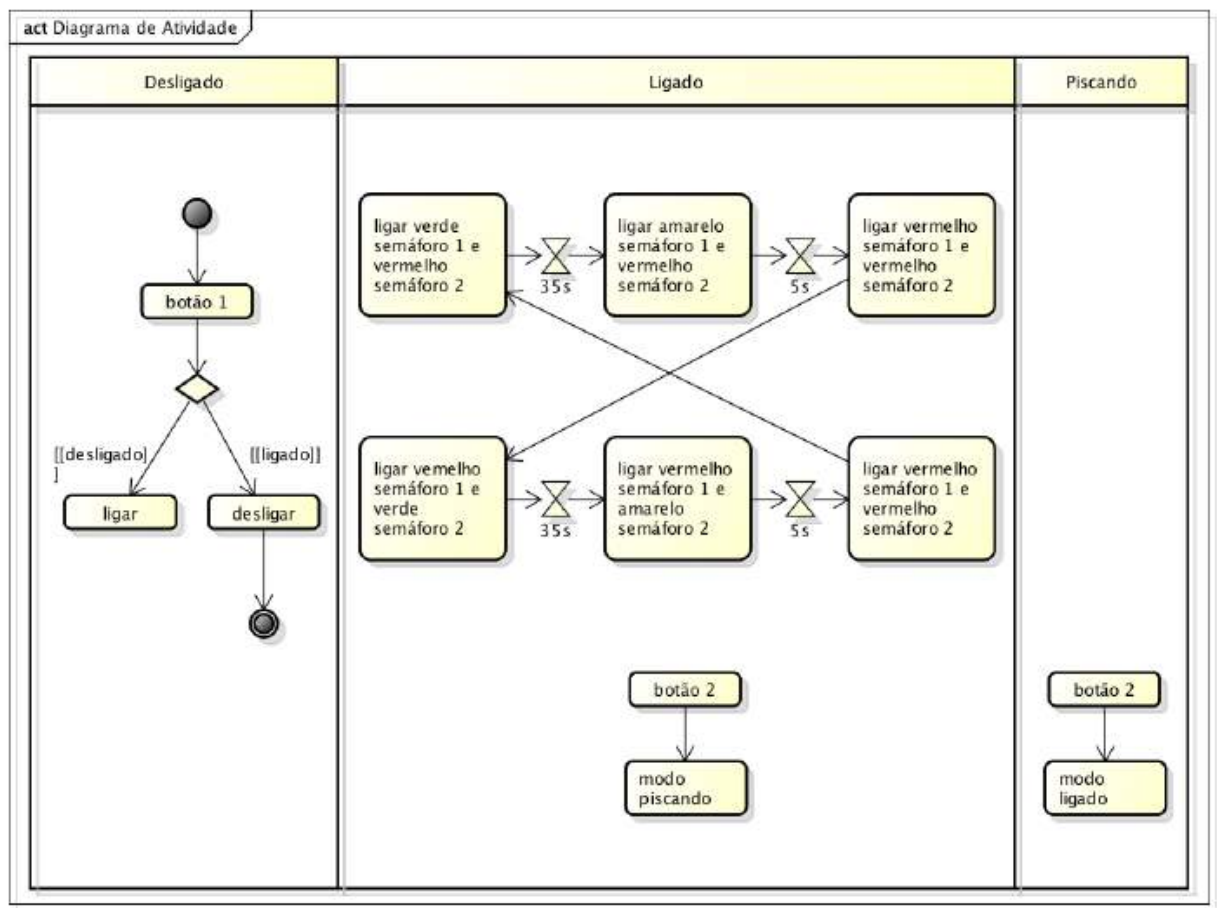

Figura 2. Diagrama de Atividades

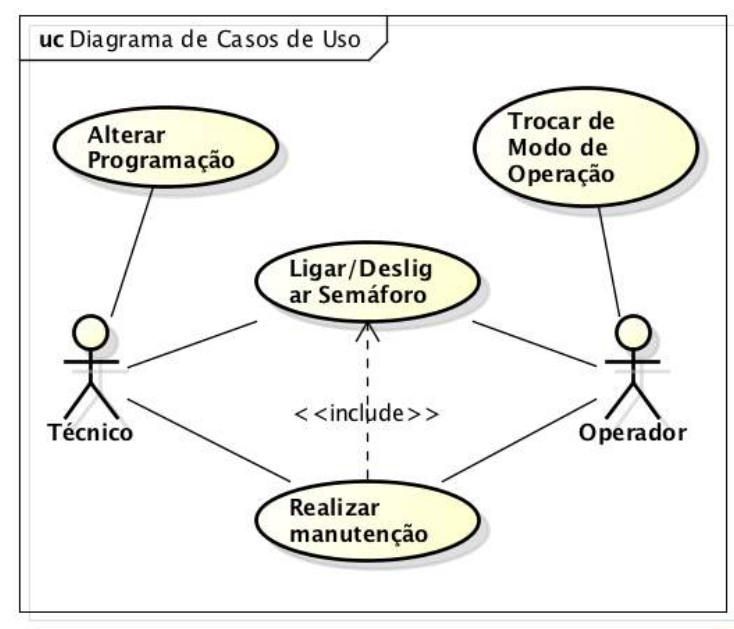

powered by Astah

Figura 3. Diagrama de Casos de Uso

equipes, mescladas em alunos que já tinham conhecimento básico da plataforma Arduino com os que não a conheciam. No total, 53 alunos utilizaram o recurso.

O processo de avaliação se iniciou com um treinamento geral que introduziu a plataforma e as principais funcionalidades do Arduino IDE. Em seguida, os alunos foram divididos em grupos para a etapa de replicação dos projetos disponibilizados. Após a montagem e teste destes projetos de acordo com seus diagramas, os alunos foram incumbidos do desenvolvimento de um projeto próprio, seguindo os moldes dos projetos presentes no recurso educacional disponibilizado. Terminadas as etapas práticas, os alu- 


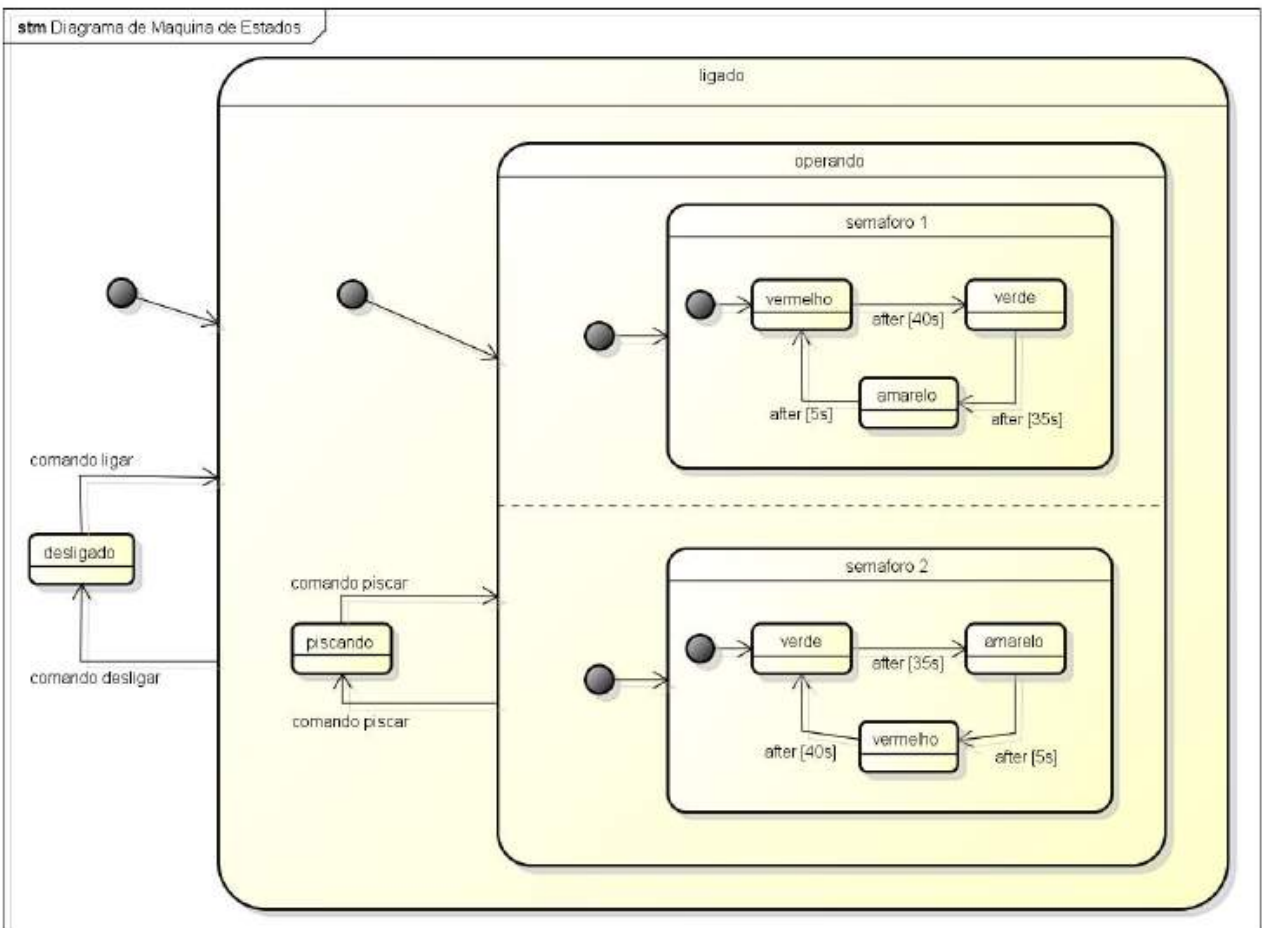

Figura 4. Diagrama de Máquina de Estados

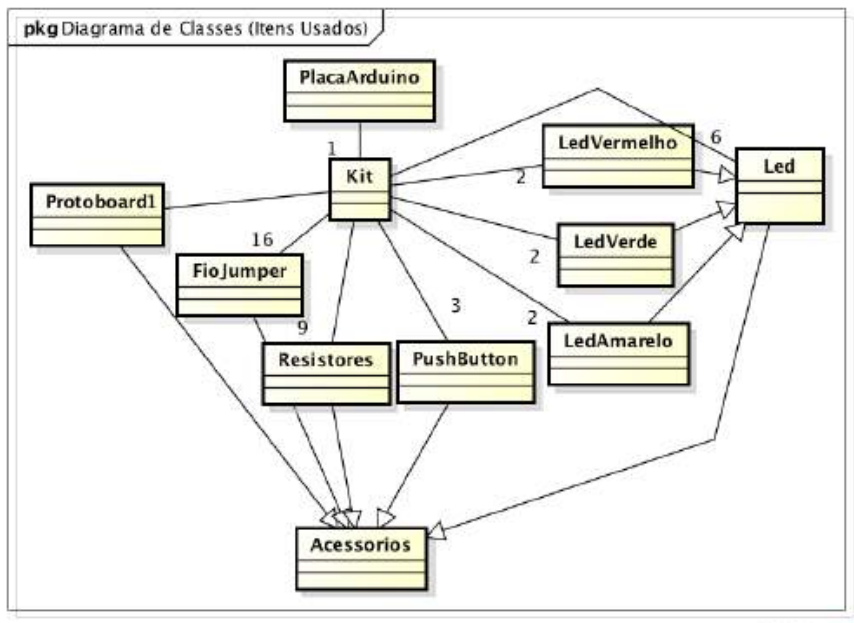

Figura 5. Diagrama de Classes

nos realizaram a identificação de defeitos encontrados nos projetos disponibilizados (nos códigos, esquemáticos e diagramas), e responderam a um questionário pós-estudo, cujos detalhes são apresentados na Seção 4.

Durante a aplicação do recurso, os alunos foram instruídos a seguirem um processo de desenvolvimento, conforme ilustrado na Figura 6. Tal processo, que pode ser usado em futuras replicações, os auxiliou no uso correto dos diagramas UML e na reflexão sobre o entendimento dos artefatos. Acredita-se que o processo também auxiliou na prevenção de danos causados aos componentes do kit Arduino. 


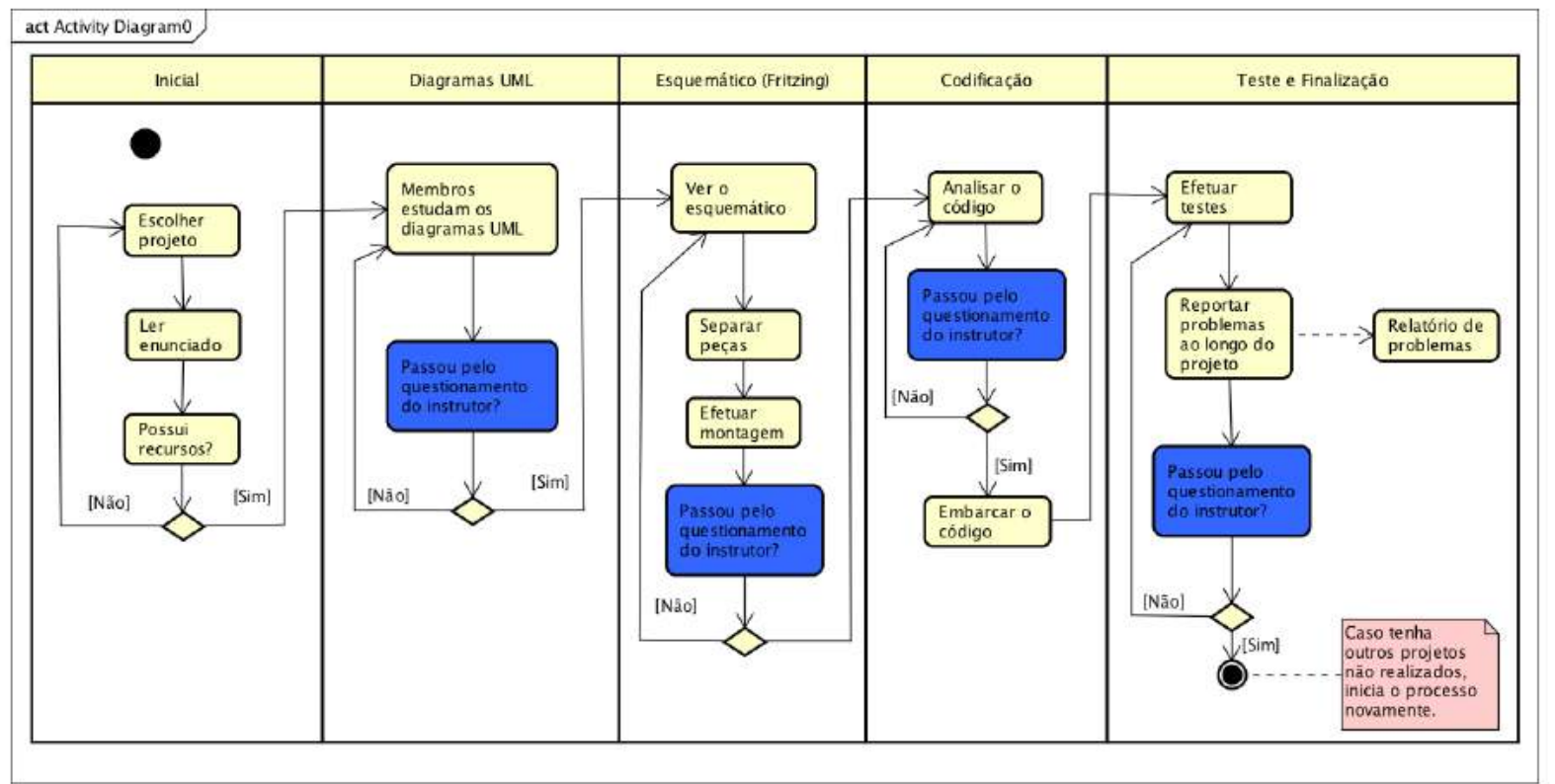

Figura 6. Processo de Uso do Recurso Educacional

\section{Instrumento de Avaliação do Recurso}

Com o intuito de analisar a abordagem utilizada e avaliar os pontos que devem ser melhorados, um questionário foi aplicado logo após a conclusão do processo apresentado na Figura 6. O questionário continha 15 questões com opções de resposta em escala Likert, utilizada para respostas psicométricas em pesquisas de opinião, cujos valores são definidos de um a cinco. O valor um representa completo desacordo ou negação, enquanto que o valor cinco representa completo acordo ou aprovação. O questionário também conta com uma questão aberta em que o aluno poderia dar sugestões e opiniões. As questões e suas respostas são apresentadas na Tabela 1. As respostas às Questões de 1 a 8 e de 9 a 15 são apresentadas na forma de gráficos nas Figuras 7 e 8, respectivamente.

Segundo a Questão 2 da avaliação conduzida, os alunos entenderam que o recurso educacional permitiu consolidar os conhecimentos de UML inicialmente aprendidos em teoria. Este resultado está em consonância com o objetivo esperado de uso do recurso ao possibilitar que o estudante treine sua habilidade de interpretar os artefatos da UML e verifique o resultado de suas interpretações no projeto embarcado em Arduino. O resultado da Questão 1 indica que o recurso auxiliou na aquisição de novos conhecimentos com a criação de novos diagramas após a atividade de treinamento. A aplicação da teoria em novos problemas gera soluções não previstas anteriormente nas aulas teóricas e, por consequência, produz novos conhecimentos.

Os resultados das Questões 3 a 6 indicam uma acentuada aceitação do recurso educacional e interesse no uso e prática individual. As respostas das Questões 7 e 8 indicam que os alunos entendem, na prática, a importância do uso de UML e esquemáticos em projetos embarcados. Esta última indicação é um fator pedagógico importante, dado que o aprendizado apenas teórico pode não transmitir devidamente a importância do conteúdo ministrado ao aluno. As respostas às Questões 9 a 15 evidenciam que o recurso educacional está, de uma forma geral, apropriado aos seus objetivos. No entanto, melhorias 


\begin{tabular}{|l|l|l|l|l|l|}
\hline Questões & CT & CP & I & DP & DT \\
\hline $\begin{array}{l}\text { 1: O recurso educacional utilizado permitiu adquirir novos } \\
\text { conhecimentos? }\end{array}$ & 44 & 6 & 1 & 2 & 0 \\
\hline $\begin{array}{l}\text { 2: O recurso educacional utilizado permitiu consolidar co- } \\
\text { nhecimentos já existentes? }\end{array}$ & 40 & 11 & 2 & 0 & 0 \\
\hline $\begin{array}{l}\text { 3: O recurso educacional deve ser utilizado novamente } \\
\text { nesta disciplina? }\end{array}$ & 46 & 1 & 3 & 2 & 1 \\
\hline 4: O recurso educacional foi aplicado no tempo adequado? & 25 & 19 & 3 & 4 & 2 \\
\hline 5: Esta atividade deveria ser feita individualmente? & 5 & 12 & 8 & 9 & 19 \\
\hline $\begin{array}{l}\text { 6: A configuração em equipes foi adequada para esta ativi- } \\
\text { dades? }\end{array}$ & 34 & 13 & 3 & 2 & 1 \\
\hline $\begin{array}{l}\text { 7: Os diagramas em UML foram essenciais para a execução } \\
\text { dos projetos? }\end{array}$ & 25 & 20 & 6 & 1 & 1 \\
\hline $\begin{array}{l}\text { 8: Os esquemáticos da ferramenta Fritzing foram essenciais } \\
\text { para o desenvolvimento dos projetos? }\end{array}$ & 42 & 9 & 1 & 1 & 0 \\
\hline $\begin{array}{l}\text { 9: Os códigos fonte em C foram essenciais para a execução } \\
\text { dos projetos? }\end{array}$ & 44 & 6 & 1 & 2 & 0 \\
\hline $\begin{array}{l}\text { 10: O processo seguido para a execução dos projetos foi } \\
\text { adequado? }\end{array}$ & 46 & 3 & 3 & 1 & 0 \\
\hline 11: Novos projetos devem ser adicionados ao recursos? & 38 & 10 & 5 & 0 & 0 \\
\hline 12: Os diagramas UML precisam ser melhorados? & 38 & 9 & 6 & 0 & 0 \\
\hline 13: Os esquemáticos em Fritzing precisam ser melhorados? & 2 & 19 & 15 & 6 & 11 \\
\hline 14: Os códigos-fonte em C precisam ser melhorados? & 3 & 11 & 13 & 8 & 18 \\
\hline $\begin{array}{l}\text { 15: Os artefatos (códigos, diagramas) em Inglês complica- } \\
\text { riam o entendimento? }\end{array}$ & 1 & 13 & 19 & 11 & 9 \\
\hline
\end{tabular}

Tabela 1. Respostas ao Questionario: CT - Concordam Tortalmente; CP - Corcordam Parcialmente; I - Indiferentes; DP - Discordam Parcialmente; DT - Discordam Totalmente

no recurso educacional ainda são necessárias. Por exemplo, escolhas mais didáticas podem ser propostas para determinadas abstrações e diferentes visões/versões de um mesmo diagrama podem ser introduzidos.

Em relação a questão aberta, grande parte dos alunos reportaram uma boa experiência (principalmente em relação ao caráter prático dos projetos) e advogaram pela continuidade da atividade na disciplina. Alguns alunos questionaram o tempo da atividade, sugerindo um maior tempo para desenvolver os projetos ou mesmo uma disciplina focada neste conteúdo. Outros destacaram que a atividade foi o primeiro contato com a plataforma Arduino, fato que aumentou sua motivação. Um aluno questionou a adequação de alguns diagramas (por exemplo, o diagrama de classe), uma vez que tal representação nem sempre se traduz em classes no código. Outro sugeriu a inclusão de projetos maiores e mais complexos; na realidade, o recurso possui um projeto de um robô vigilante que não foi aplicado devido a insuficiência de componentes para todos os grupos. A ideia é que esse e outros projetos mais complexos possam ser aplicados no futuro. Por fim, a divisão das equipes foi um ponto de controvérsia: uns gostaram de trabalhar com outros colegas, enquanto que outros preferiam colaborar com seus amigos. O número de participantes na 


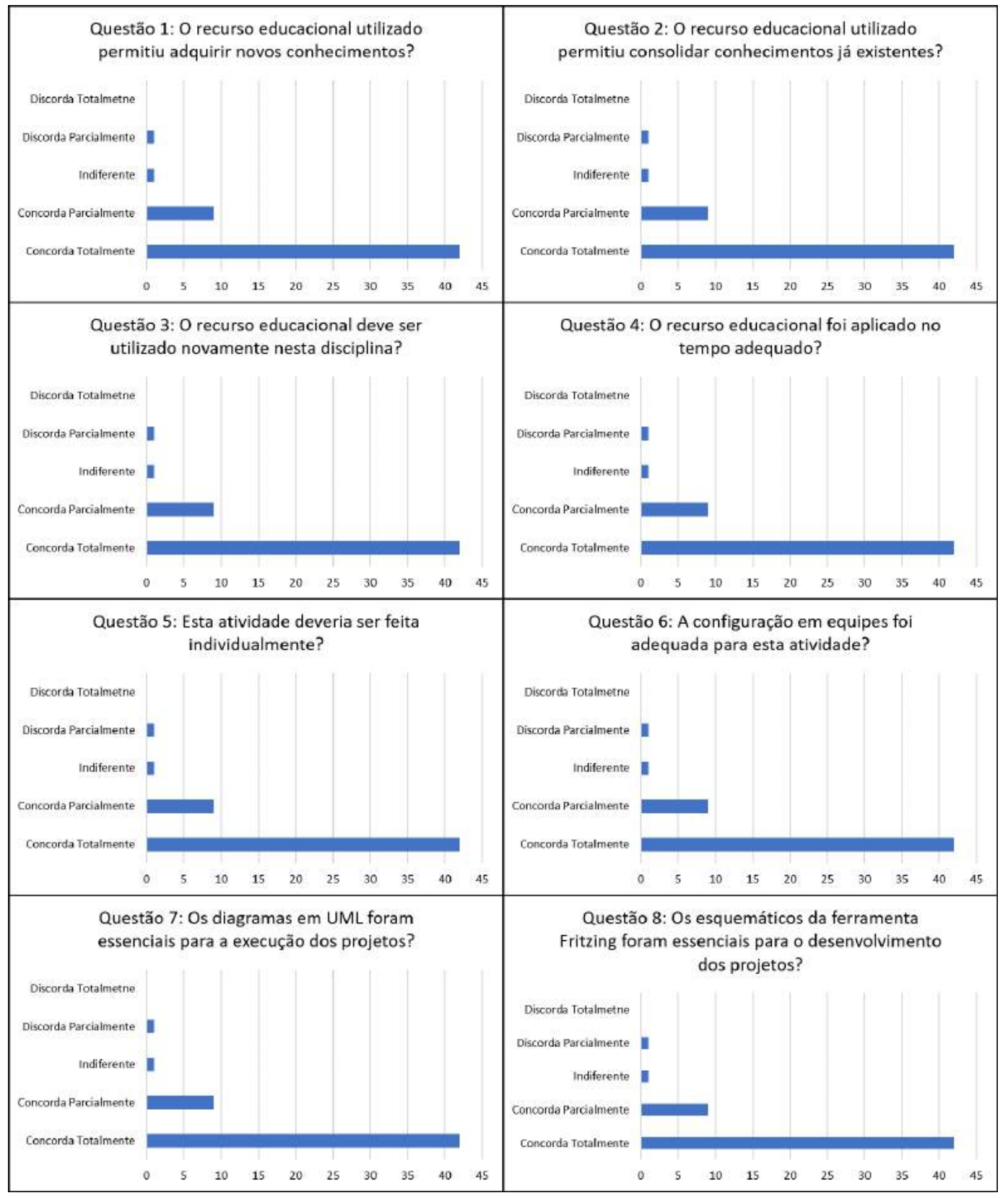

Figura 7. Respostas das Questões de 1 a 8

equipe e a necessidade de fazer todos os projetos em equipe também foram outros pontos ponderados.

\section{Conclusão}

Recursos e exemplos da literatura, usados em aula para aproximar a teoria da prática em Engenharia de Software, geralmente enfatizam sistemas de informação. Tais temas não representam o foco principal em alguns cursos de Bacharelado em Engenharia. A abordagem apresentada visou a utilizar temas de maior aderência - como sistemas embarcados e Arduino - para estes cursos na criação de um recurso educacional aberto.

Uma vez que a avaliação do recurso proposto foi predominantemente positiva, 


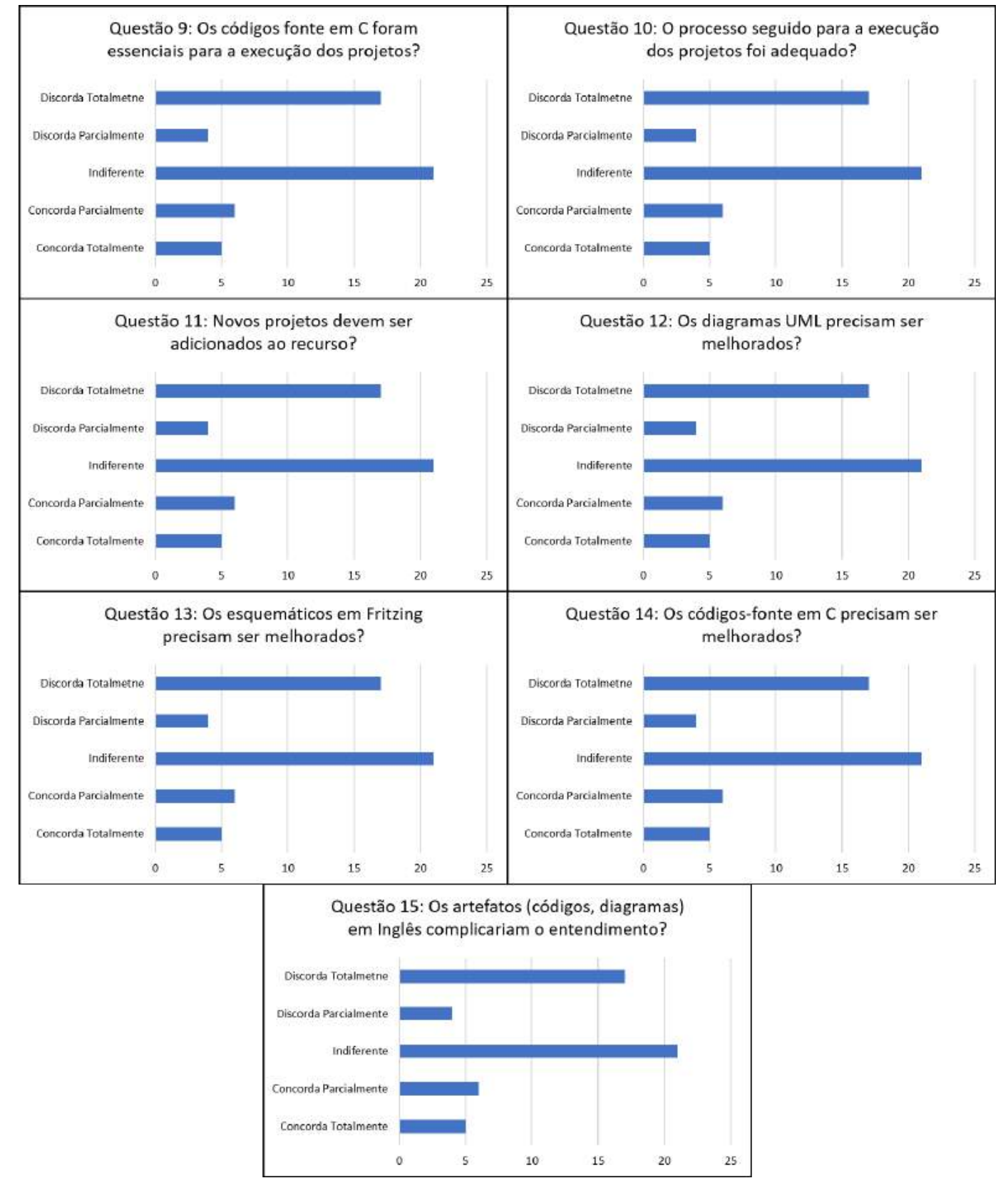

Figura 8. Respostas das Questões de 9 a 15

espera-se que a proposta apresentada possa contribuir no apoio ao ensino da disciplina de análise e projeto de software em cursos de Bacharelado em Engenharia, cujos recursos são, ainda, pouco focados em suas distintas características. Como trabalhos futuros, pretende-se investigar a disponibilização dos artefatos em outro idioma e a extensão do recurso a outras fases da Engenharia de Software, tais como requisitos, teste e processos.

Agradecimentos O projeto apresentado foi financiado pelos editais 32/2014 e 15/2015 -PROGRAD/UTFPR. A. T. Endo é parcialmente financiado pelo CNPq (445958/2014-6). 


\section{Referências}

Arduino (2017). The arduino platform. Online em: https://www.arduino.cc/.

Avila, L.; Bernardini, F. C. M. P. (2016). O uso de robótica para aprendizado de programação integrando alunos de educação básica e ensino superior. In Workshop sobre Educação em Computação, pages 2277-2283.

Brock, J. D., Bruce, R. F., and Reiser, S. L. (2009). Using arduino for introductory programming courses. Journal of Computing Sciences in Colleges, 25(2):129-130.

Cavalcante, M. M., Silva, J. L. d. S., Viana, E. C., and Dantas, J. R. (2014). A plataforma arduino para fins didáticos: estudo de caso com recolhimento de dados a partir do plx-daq. In Workshop sobre Educação em Computação.

Damian, D., Hadwin, A., and Al-Ani, B. (2006). Instructional design and assessment strategies for teaching global software development: a framework. In International conference on Software engineering, pages 685-690. ACM.

de Souza, A. R., Paixão, A. C., Uzêda, D. D., Dias, M. A., Duarte, S., and de Amorim, H. S. (2011). A placa arduino: uma opçao de baixo custo para experiências de fisica assistidas pelo pc. Revista Brasileira de Ensino de Fisica, 33(1).

Duschl, K., Obermeier, M., and Vogel-Heuser, B. (2014). An experimental study on uml modeling errors and their causes in the education of model driven plc programming. In IEEE Global Engineering Education Conference (EDUCON), pages 119-128.

Freitas, O. (2009). Equipamentos e materiais didáticos. Universidade de Brasília.

Grover, R., Krishnan, S., Shoup, T., and Khanbaghi, M. (2014). A competition-based approach for undergraduate mechatronics education using the arduino platform. In 4th Interdisciplinary Engineering Design Education Conference (IEDEC), pages 78-83.

Larman, C. (2012). Applying UML and Patterns: An Introduction to Object Oriented Analysis and Design and Interative Development. Pearson Education India.

Mattos, G. d. O., da Silva, D. R. D., and Moreira, J. A. (2015). A utilização de kits de robótica como ferramenta para o ensino de programação à meninas do ensino médio. In Workshop sobre Educação em Computação, pages 2277-2283.

Melo, R. M., Filho, I. J. M., Feliciano, F. D. O., Carvalho, R. S., Brito, J. A., and Gomes, A. S. (2014). Using arduino as pedagogical strategy in learning logic programming. In Iberian Conference on Information Systems and Technologies, pages 1-5. IEEE.

Pinto, M. d. C., Elia, M., and Sampaio, F. (2012). Formação de professores em robótica educacional com hardware livre arduino no contexto um computador por aluno. In Workshop de Informática na Escola, volume 1.

Souza, M. M., Resende, R. F., Prado, L. S., Fonseca, E. F., Carvalho, F. A., and Rodrigues, A. D. (2010). Sparse: Um ambiente de ensino e aprendizado de engenharia de software baseado em jogos e simulação. In Simpósio Brasileiro de Informática na Educação.

Yamaguti, M. H.; de Oliveira, F. M. (2016). Ages - um espaço interdisciplinar, baseado em projetos, para aprendizagem de engenharia de software. In Congresso da Sociedade Brasileira de Computação - Curso de Qualidade, pages 329-332. 